\title{
CFD ANALAYSIS OF FLOW THROUGH A CONICAL EXHAUST DIFFUSER
}

\author{
R.Prakash ${ }^{1}$, D.Christopher ${ }^{2}$, K.Kumarrathinam ${ }^{3}$ \\ ${ }^{l}$ Assistant Professor, Department of Mechanical Engineering, SSN College of Engineering, Tamil Nadu, India \\ ${ }^{2}$ Student, Department of Mechanical Engineering, SSN College of Engineering, Tamil Nadu, India \\ ${ }^{3}$ Student, Department of Mechanical Engineering, SSN College of Engineering, Tamil Nadu, India
}

\begin{abstract}
The exhaust diffuser of a fluid machine such as a gas turbine recovers static pressure by decelerating the flow and converting kinetic energy into pressure energy. It is hence a critical component in a turbo machine environment and plays a pivotal role in determining the performance of a turbo machine. Therefore, if the diffuser design is optimized for maximum pressure recovery, an increase in efficiency of the fluid machine can be brought about. Computational fluid dynamics (CFD) analysis was performed on diffusers with different half cone angles and based on the results obtained the geometry that yielded the maximum pressure recovery was identified. The diffuser with the optimized geometry was then fabricated and tested. The experimental value of the diffuser pressure recovery coefficient was calculated and compared with the theoretical value.
\end{abstract}

Keywords: Diffuser, Computational Fluid Dynamics, Pressure Recovery

\section{INTRODUCTION}

The exhaust diffuser of the gas turbine is used to reduce the velocity of the working fluid discharged from the turbine and hence increase its pressure. This increases the pressure ratio across the turbine. Also, since the diffuser increases the pressure of the working fluid, the pressure gradient at the diffuser exit reduces. The fluid can, therefore be expelled from the gas turbine unit with more ease than in the absence of a diffuser. This is because in a system without a diffuser, the atmospheric pressure tends to push the fluid back into the turbine, causing backflow and resulting in a considerable drop in turbine performance. The diffuser assists in this expulsion of exhaust gases, thereby reducing the turbine work spent on pushing the gases out. Hence, the useful work of the turbine increases, thereby increasing the efficiency of the turbo machinery system.

Over the past decade, there has been a sustained interest in the analysis of exhaust diffusers owing to the effect it has on the overall efficiency of a fluid machine system and hence considerable work on has been done in this sphere. Both numerical and experimental investigation has been performed on diffusers to a great extent with the aim of improving the pressure recovery and consequently the efficiency of the turbo machine system. The use of computational fluid dynamic analysis is also rapidly gaining importance with regard to work in this sphere. The accuracy of the results obtained has increased considerably with the advent of computational methods. CFD analysis of industrial gas turbine exhaust diffuser was carried out by Kouichi Ishizaka, Susumu Wakazono [1]. The purpose of this study was to improve the performance of exhaust diffuser in consideration of the distortion of the flow of the turbine exit, the diffuser performance in the big flow rate and to verify of CFD accuracy. Predicted turbine exit flow and diffuser pressure recovery was in reasonably good agreement with the measured data. Singh et al [2] conducted CFD studies of annular diffuser with different geometries but same inlet cone angle and reported that the performance of the annular diffuser having parallel diverging hub and casing was improved upon the introduction of swirl. SJ Kline, DE Abbott, RW Fox[3] showed that diffuser efficiency is determined by global parameters and equations which correlate static pressure and total pressure at the inlet and the outlet. Baskharone.E.A [4] developed a Finite element model of turbulent flow in annular exhaust diffusers of gas turbine engines and verified the results using a set of experimental data. Assessment of the results provided evidence of the model capability as an accurate predictive tool in the diffuser detailed design phase. The use of computational analysis has also allowed the inclusion of parameters such as turbulence, which involving solving highly complex equations and the effect of which are otherwise very difficult to be taken into consideration. A.C. Benim, W. Zinser [5] applied a finite element method is to turbulent confined flow problems adopting a primitive-variables formulation. The turbulence state is defined by a $k-\varepsilon$ model of turbulence. A general overview of the turbulent flow in a conical diffuser was presented by R. S. Azad and S. Z. Kassab [6]. The key aspects for the reliable CFD modelling of exhaust diffusers are addressed and an automated procedure for a time- and resource-efficient and accurate prediction of complex diffuser configuration is presented by V. Vassiliev, S. Irmisch, S. Florjancic[7].P.A.C. Okwuobi and R.S.Azad [8] performed an experimental study on the structure of turbulence in a conical diffuser and showed that the rate of turbulent energy production approximately reaches a maximum value at the edge of the wall layer extending to the point of maximum fluctuation. The turbulent kinetic energy balance indicated that the magnitude of the energy convective diffusion due to kinetic and pressure effects was comparable with that of the 
energy production. Hoffman and Gonzalez [9] proved that increasing inlet turbulence intensity increases the pressure recovery of the diffuser. S.W. Armfield, C.A.J.Fletcher [10] presented a comparison of $k-\varepsilon$ and algebraic Reynolds stress models for swirling diffuser flow, demonstrating that the standard $k-\varepsilon$ model gives poor prediction of the mean flow, and it was necessary to use the other forms of the $k-\varepsilon$ model. B. K. Sultanian, S. Nagao and T. Sakamoto[11] showed that the applied Navier-Stokes method is capable of predicting complicated gas turbine exhaust system flows for design applications.

The aim of this paper is to identify by means of computational fluid dynamic analysis, the diffuser half cone angle which provides maximum pressure recovery, to fabricate the diffuser of optimized geometry and to perform tests on this diffuser to experimentally validate the simulation results.

\section{CFD MODELING}

$3 \mathrm{D}$ models of the diffuser with half cone angles of $5^{0}, 7^{0}, 10^{\circ}$, $12^{\circ}$ and constant area ratio were created using CATIA V5R17. The relevant dimensions were chosen from literature [8], based on which the area ratio was found to be 3.08. The dimensions of the diffuser model are shown in Figure 1. The model was imported into ANSYS Workbench where meshing, preprocessing, solving and post processing were performed. The mesh was generated in the meshing environment of ANSYS workbench. The physics preference was selected as CFD and solver preference was selected as FLUENT. The mesh settings were selected in such a way that a balance was struck between accuracy and computing speed. The relevance was set to zero and relevance center was set at medium. Curvature based mesh refinement was used. The initial size seed option was set to active assembly, as the model consists of only a single geometry. The smoothing was set to medium, in order to have an optimized number of smoothing iterations to improve mesh smoothness. A slow transition rate is selected to ensure a gradual growth rate between adjacent elements. The mesh generated consisted of 8967 nodes and 23640 elements. The generated mesh is shown in Figure 2. The physics setup was done in FLUENT - a fluid flow analysis system in the ANSYS workbench. This package is widely used for simulation, visualization, and analysis of fluid flow, heat and mass transfer, flow-induced noise and chemical reactions. It is a vital part of the computer-aided engineering (CAE) process for companies around the world and is deployed in many manufacturing industries. Double precision solver was selected as the diffuser consists of sections of disparate length and this type of geometry may benefit from the use of this solver even though consumption of system resources may be high. Serial processing option was selected as simulation was being done a single machine only. The mesh generated was first checked for inconsistencies. A pressure based steady state solver was used as the flow was assumed to be incompressible and the velocity and pressure did not vary with time. The $\mathrm{k}-\varepsilon$ turbulence model was used for this simulation owing to its robustness and its ability to strike a balance between computational accuracy and consumption of computing resources. Out of the various types of this model, the realizable type was selected owing to its suitability for flows involving pressure gradients. In the near-wall region, the turbulent boundary layer is very thin and the solution gradients are very high, but accurate calculations in the near-wall region are paramount to the success of the simulation. Hence, a very fine mesh to resolve this region, but it is usually very expensive for industrial CFD applications. As standard wall functions are generally sufficient for simple flows with moderate pressure gradients and separation, it was chosen. Non equilibrium wall functions are generally used for flows with large and abrupt separation and pressure changes. Enhanced wall treatment is used to generate a very finely meshed wall region and provide more accurate solutions. The working fluid is chosen to be air and its properties are listed in Table 1. Boundary conditions specify the flow variables on the boundaries of the physical model. They are, therefore, a critical component of the simulation and it is important that they are specified appropriately. A velocity inlet boundary condition was used for the diffuser inlet. A fluid velocity of 45 $\mathrm{m} / \mathrm{s}$ was specified as obtained from literature [8]. The direction of velocity was chosen to be normal to the boundary. The reference frame was arbitrarily chosen as absolute. The reference frame comes into play only when the problem involves a moving boundary and does not affect a fixed boundary in any way. Turbulent intensities vary depending on the application. For example, a high turbulence level (5\% to $20 \%$ ) is used for high speed flows in complex rotor dynamic machines. A medium turbulence level (1\% to 5\%) is used for flows through pipes, vents, nozzles and diffusers. Therefore a turbulence intensity of $2.5 \%$ was used in this simulation. Low turbulence levels (below 1\%) are used for flows originating from fluid that stands still like external flow across cars. The hydraulic diameter at the inlet was the same as the diameter of the inlet section of the diffuser $(57 \mathrm{~mm})$. A pressure outlet boundary condition was used for the diffuser outlet. As, the diffuser exits to the atmosphere, the pressure was set to 101325 Pascal. Backflow turbulence intensity was neglected. The hydraulic diameter at the exit was the same as the diameter of the diffuser outlet $(100 \mathrm{~mm})$. The wall was assumed to be smooth. Hence roughness height and roughness constant were set to zero. As the diffuser is stationary, wall motion was set to stationary. Also, the fluid was allowed to slip over the surface of the wall in order to allow flow. As mentioned before, a pressure based solver was used. The solver scheme was set to coupled where the momentum and continuity equations are solved simultaneously thus leading to a faster convergence rate, compared to the segregated scheme where the equations are solved sequentially. FLUENT stores discrete values of the scalar at the cell centers. However, face values are required to solve the equations and these must be interpolated from the cell center values. This is accomplished using an upwind scheme. Upwinding means that the face value is derived from quantities in the cell upstream relative to the direction of the normal velocity. In this simulation the first order upwind scheme has been chosen for turbulence, momentum and energy, as first order accuracy was deemed sufficient. The second order scheme provides more accurate solutions and the power law scheme interpolates the face value using a solution to a one dimensional equation. Solution monitors allow dynamic monitoring of convergence. At the end of every solver iteration, the residual sum for each of the 
conserved variables is computed and stored. The convergence can be allowed to terminate once the residuals attain the user defined values. The parameters such as continuity, components of velocity and momentum are assigned residual monitors of $1 \mathrm{e}-11$ and the turbulence parameters $\mathrm{k}$ and epsilon are assigned residual monitors of $1 \mathrm{e}-7$. The convergence criterion is set to absolute as this setting is suited for steady state problems. Solution initialization is then done to provide fluent with initial guess values for the iterations. The initialization is computed from the inlet as the inlet is the section of the diffuser where flow begins. The FLUENT case is then checked for inconsistencies before the iterations begin. A termination criterion of 2000 iterations is set and the calculation is started. For all the simulations, the solution convergence occurred at about 400 to 450 iterations.

\subsection{Results of the CFD Analysis Performed}

The solutions obtained were then converted to plots and contours using the post processing interface of FLUENT. Figures 5.4 to 5.25 show the contours and plots obtained. The pressure plots and contours show that the static pressure remains constant along the entire length of the diffuser inlet pipe, rise sharply along the length of the diverging diffuser body and become constant along the length of the exit pipe. The velocity plots and contours depict an exactly opposite trend, owing to the conversion of kinetic energy into pressure energy. Also, it can be seen that the centerline velocity is higher than the velocity at the boundary due to friction effects at the boundary layer. The plots and contours of turbulence parameters such as turbulence intensity, turbulence dissipation rate and turbulent kinetic energy all undergo a rapid change at the point of divergence of the diffuser. These plots are shown only for the diffuser with a half cone angle of $7^{0}$ as this diffuser provided the maximum value of CPR. This behavior can be attributed to the fact that the sudden change in area of cross section leads to the formation of eddies leading to increased turbulence. Table 2 presents a summary of the various parameters obtained from the analysis and those calculated from this data. The coefficient of pressure recovery (CPR) is a measure of the recovery of static pressure. A high CPR value indicates that a large proportion of the available dynamic head has been converted to pressure head, hence leading to a large pressure recovery. CPR was calculated using the formula:

$$
\mathrm{CPR}=\left(\mathrm{P}_{\mathrm{x}}-\mathrm{P}_{\mathrm{i})} / \mathrm{q}_{\mathrm{i}}\right.
$$

Where $P_{x}$ is the average static pressure at any station $x, P_{i}$ is the static pressure at the diffuser inlet and $q_{i}$ is the dynamic head available at the inlet of the diffuser. The values of static pressure were obtained by listing the solution to file and choosing values at points on the diffuser located at a distance of $40 \mathrm{~mm}$ from the inlet and the exit. This was done as the measuring stations used while conducting experiments were situated at the same distances from the inlet and exit sections.

\section{EXPERIMENTAL SETUP}

From the CFD analysis performed on diffusers of various geometries, it was found that the diffuser with a half cone angle of $7^{0}$ provided the maximum pressure recovery. Hence this geometry was chosen for fabrication and subsequent testing. The diffuser was fabricated using an aluminum sheet of $2 \mathrm{~mm}$ thickness. The material selection was done in such a way that a balance between reduced weight and sufficient strength could be obtained. The area ratio of the fabricated diffuser was 3.08 , but certain other dimensions were modified in order to enable the mounting of the diffuser onto the experimental test rig. The constant area ratio ensures that the half cone angle remains unchanged, thereby replicating the conditions used for CFD analysis. Figure 20 shows the various dimensions of the fabricated diffuser and Figure 21 shows the fabricated diffuser. The experimental setup consisted of the fabricated diffuser, a centrifugal blower to provide the necessary discharge of fluid and a Pitot tube for pressure measurements. Figure 22 shows the experimental setup. A centrifugal blower was used instead of a wind tunnel to provide the necessary fluid discharge. The diffuser was mounted to the discharge end of the blower by means of the flange at the inlet section of the diffuser. Table 3 shows the specifications of the blower used. A Pitot tube with a digital manometer was used to measure the static and dynamic pressures at the two stations. Two holes were drilled at a distance of $40 \mathrm{~mm}$ from the inlet and exit of the diffuser to facilitate insertion of the Pitot tube. For measurement of pressure parameters, first the electronic connections were made and the digital display unit was switched on. The pressure readout was then calibrated to zero using the calibration knob. The hole drilled at measurement station 2 was sealed tightly with clay to prevent leakage of fluid. The static tube connected to the static port was removed. The instrument was then inserted into measurement station 1. After a stabilization time of 5 minutes, the stagnation pressure displayed in the digital readout was noted. The static tube was then connected and the instrument was again inserted into the measurement station and after the same stabilization time, the pressure differential or the dynamic pressure displayed in the digital readout was noted. Then, the hole drilled at measurement station 1 was sealed and the procedure was repeated to measure the static and dynamic pressure at the other station. The stabilization time allows the flow to settle, eliminating sudden changes in pressure.

\subsection{Results and Discussion}

Table 4 presents the flow parameters obtained experimentally and the data calculated from these parameters. The digital readout of the Pitot tube displays pressure parameters in millimeters of water column. Therefore, for the calculation of flow velocity, the dynamic pressure measured was converted to Pascal.

\section{COMPARISON OF THE EXPERIMENTAL AND}

\section{THEORETICAL VALUE OF PRESSURE RECOVERY COEFFICIENT}

Computational analysis was performed on the geometry of the fabricated diffuser. The analysis was similar to the one performed earlier except that the geometry and input conditions were adjusted to match the experimental 
conditions. Figures 29 to 32 show the contours and plots obtained. Table 5 presents the data obtained from this analysis and Table 6 presents the comparison between the theoretical and experimental values of pressure recovery coefficient. The deviation between the experimental and theoretical values of pressure recovery coefficient can be minimized by carrying out certain improvements to the experimental setup. They include the use of a wind tunnel for better flow stabilization, creating swirl induced turbulence at the diffuser inlet, making the internal walls of the diffuser smooth to reduce friction and preventing leakage of fluid through the diffuser wall.

\section{CONCLUSION}

Computational analysis was performed on various geometries of diffusers and their theoretical pressure recovery coefficients were calculated using the data obtained. It was found that the diffuser with a half cone angle of $7^{0}$ provided the maximum recovery of static pressure, therefore proving to be the most efficient. A diffuser with this geometry was fabricated and was tested. Using the data collected, the experimental value of pressure recovery coefficient was calculated. The values of pressure recovery coefficient were compared and it was found that the experimental value was in close agreement to the theoretical value.

\begin{tabular}{|llll|}
\hline Nomenclature & & \\
$\mathrm{CFD}$ & Computational fluid dynamics & $\mathrm{V}$ & Volts \\
$\mathrm{CPR}$ & Coefficient of pressure recovery & $\mathrm{Hz}$ & Hertz \\
$\mathrm{P}_{\mathrm{x}}$ & Average static pressure at any station $\mathrm{x}$ & $\mathrm{HP}$ & Horsepower \\
$\mathrm{P}_{\mathrm{i}}$ & Static pressure at inlet of the diffuser & \\
$\mathrm{q}_{\mathrm{i}}$ & Dynamic head available at the inlet of the diffuser \\
$\mathrm{k}$ & Turbulent kinetic energy & \\
$\varepsilon$ & Turbulent dissipation rate & \\
\hline
\end{tabular}

Table 1 Properties of the working fluid

\begin{tabular}{|l|l|}
\hline Density $\left(\mathrm{kg} / \mathrm{m}^{3}\right)$ & 1.225 \\
\hline Specific heat $(\mathrm{J} / \mathrm{Kg}-\mathrm{K})$ & 1006.43 \\
\hline Thermal conductivity(w/m-K) & 0.0242 \\
\hline Viscosity $(\mathrm{Kg} / \mathrm{m}-\mathrm{s})$ & $1.7894 \mathrm{e}-05$ \\
\hline
\end{tabular}

Table 2 Specifications of the blower used

\begin{tabular}{|l|l|}
\hline PARAMETERS & VALUE \\
\hline Motor power & $5 \mathrm{HP}, 3$ Phase, $440 \mathrm{~V}, 50 \mathrm{~Hz}$ \\
\hline Motor speed & $1440 \mathrm{rpm}$ \\
\hline Discharge & $30 \mathrm{~m}^{3} / \mathrm{min}$ \\
\hline Discharge duct cross section & 14306.625 sq. $\mathrm{mm}$ \\
\hline Transmission & 3 speed pulley \\
\hline Blade type & Straight radial \\
\hline
\end{tabular}

Table 3 Results obtained from CFD analysis of diffusers of various half cone angles

\begin{tabular}{|l|l|l|l|l|}
\hline \multirow{2}{*}{ PARAMETER } & \multicolumn{4}{l}{ HALF CONE ANGLE OF DIFFUSER } \\
\cline { 2 - 5 } & $5^{0}$ & $7^{0}$ & $10^{0}$ & $12^{0}$ \\
\hline Static pressure at inlet (Pa) & 100218 & 100193 & 100207 & 100253 \\
& & & & \\
\hline Static pressure at exit (Pa) & 101325 & 101325 & 101325 & 101325 \\
\hline Dynamic pressure at inlet (Pa) & 1240.31 & 1240.31 & 1240.31 & 1240.31 \\
\hline Dynamic pressure at exit (Pa) & 180.24 & 145.72 & 165.83 & 203.72 \\
\hline Inlet velocity (m/s) & 45 & 45 & 45 & 45 \\
\hline Exit velocity (m/s) & 17.23 & 15.32 & 16.41 & 18.19 \\
\hline Inlet Mach number & 0.1312 & 0.1312 & 0.1312 & 0.1312 \\
\hline Exit Mach number & 0.05 & 0.044 & 0.047 & 0.053 \\
\hline Coefficient of pressure recovery & 0.8925 & 0.9126 & 0.901 & 0.864 \\
\hline
\end{tabular}


Table 4 Flow parameters obtained experimentally

\begin{tabular}{|l|l|l|}
\hline PARAMETER & STATION 1 & STATION 2 \\
\hline Stagnation pressure (or) Total pressure (mmwc) & 25 & 22 \\
\hline Dynamic Pressure (mmwc) & 95 & 22 \\
\hline Static Pressure (mmwc) & 70 & 0 \\
\hline Velocity of fluid flow (m/s) & 39 & 18.77 \\
\hline Mach number & 0.1137 & 0.0547 \\
\hline Experimental value of CPR & 0.7368 & \\
\hline
\end{tabular}

Table 5 Data obtained from CFD analysis performed on geometry of the fabricated diffuser

\begin{tabular}{|l|l|l|}
\hline PARAMETER & INLET & EXIT \\
\hline Static pressure $(\mathrm{Pa})$ & 100534 & 101325 \\
\hline Dynamic pressure $(\mathrm{Pa})$ & 931.95 & 141.69 \\
\hline Velocity of fluid flow $(\mathrm{m} / \mathrm{s})$ & 39 & 15.21 \\
\hline Mach number & 0.1137 & 0.0443 \\
\hline Theoretical value of CPR & 0.849 & \\
\hline
\end{tabular}

Table 6 Comparison of theoretical and experimental values of CPR

\begin{tabular}{|l|l|}
\hline THEORETICAL VALUE OF CPR & 0.849 \\
\hline EXPERIMENTAL VALUE OF CPR & 0.7368 \\
\hline DEVIATION & 13.57 \\
\hline
\end{tabular}
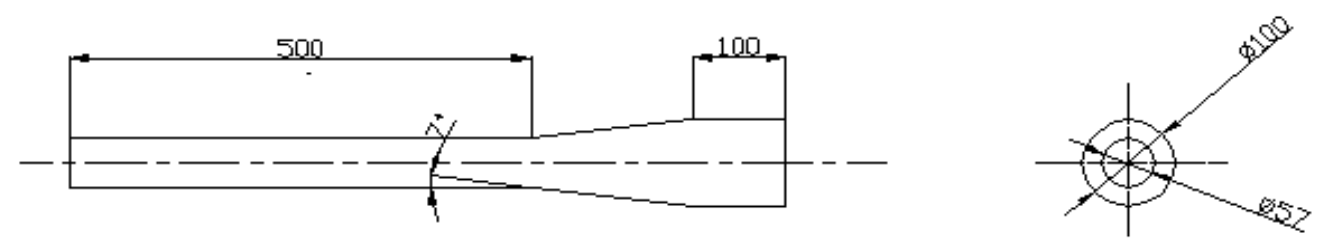

Fig 1 Dimensions of the diffuser used for CFD analysis 


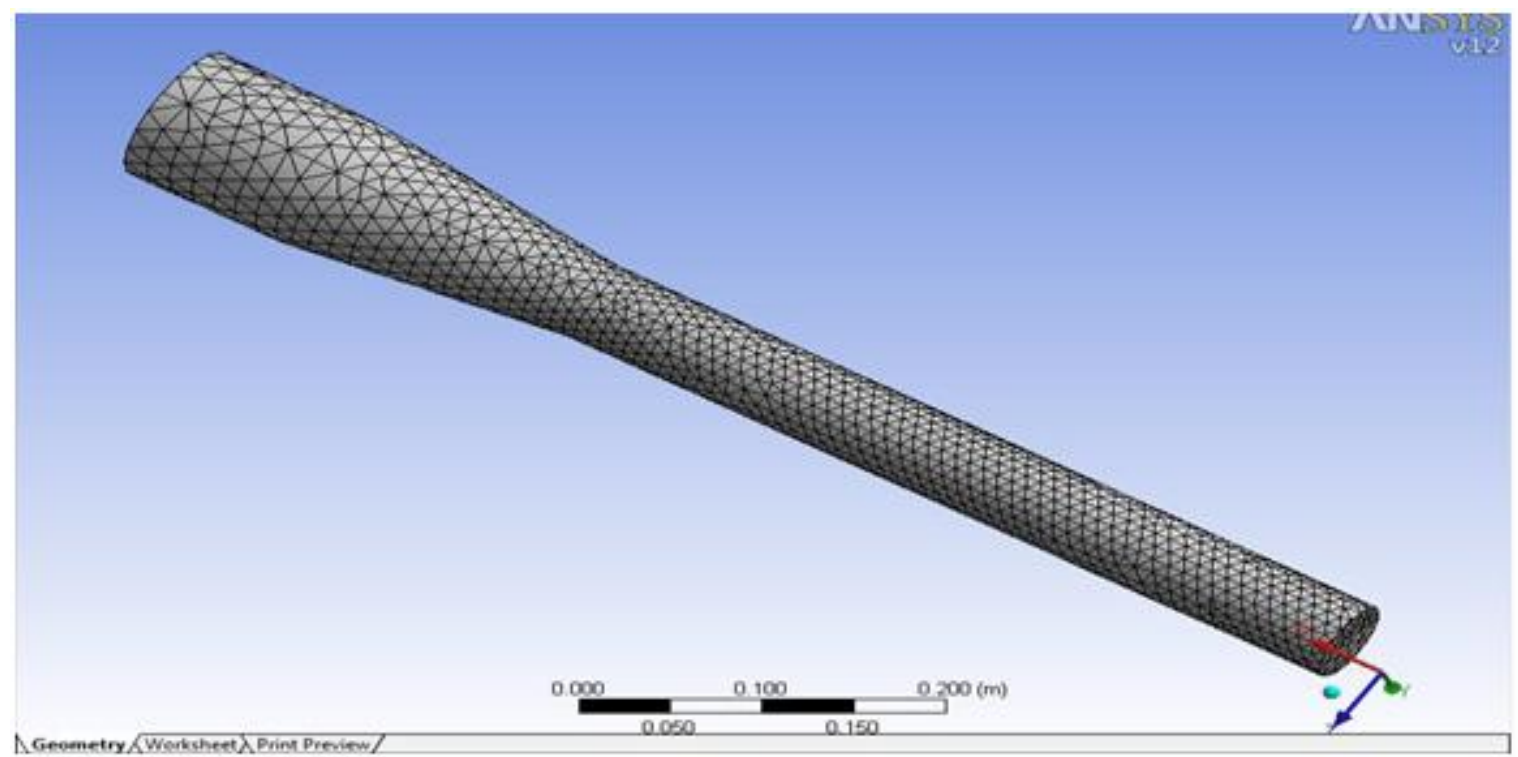

Fig 2 Generated mesh

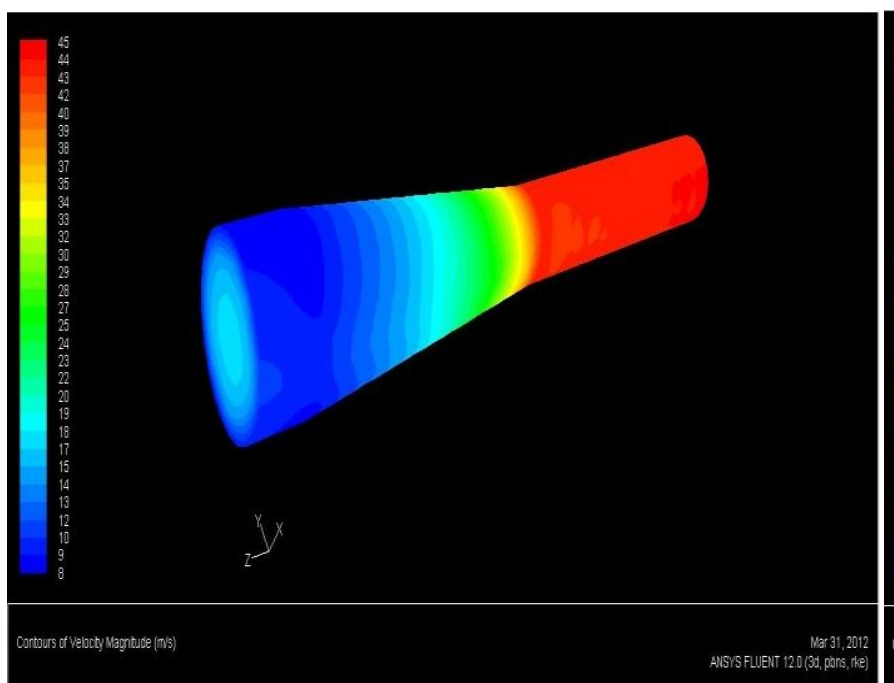

Fig 3 Contours of static pressure for diffuser of $5^{0}$ half cone Angle

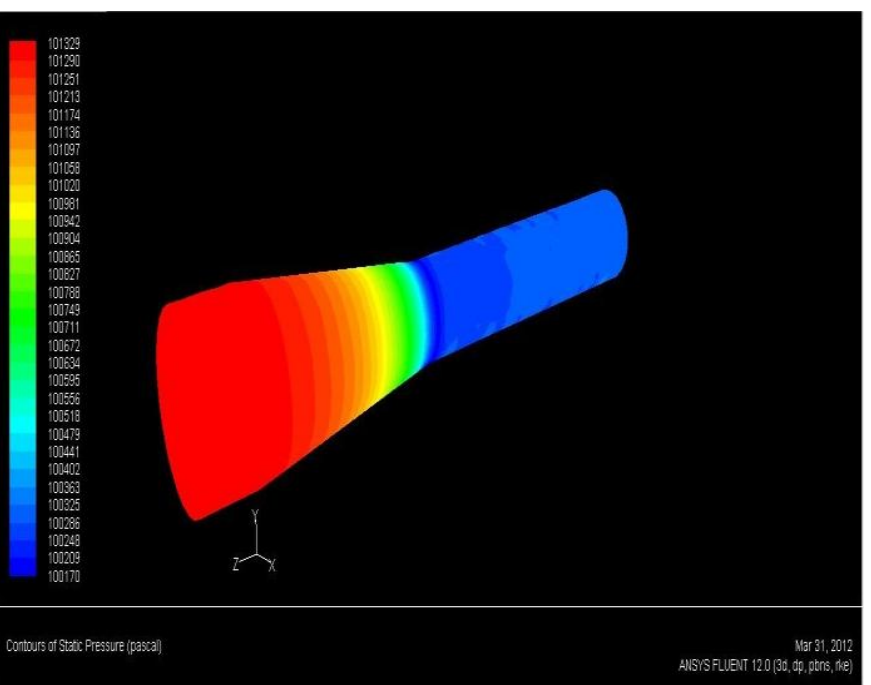

Fig 4 Contours of velocity magnitude for diffuser with $5^{0}$ half cone angle

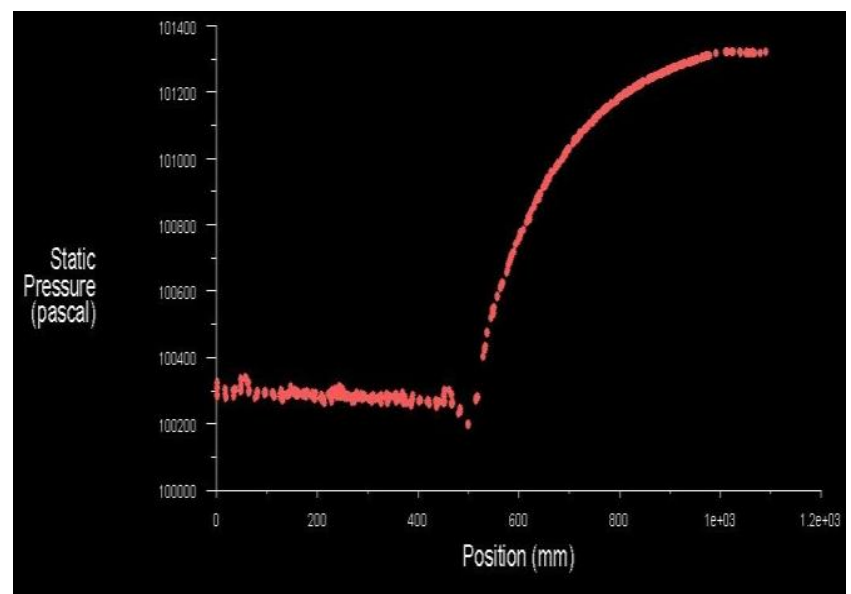

Fig 5 Variation of static pressure along the length for diffuser of $5^{0}$ half cone angle

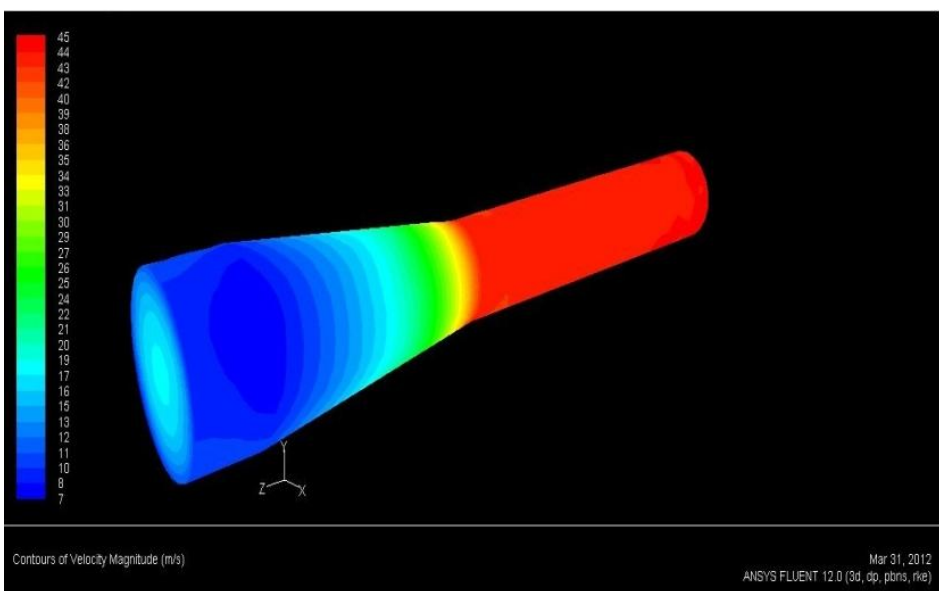

Fig 6 Variation of velocity magnitude along the length for diffuser of $5^{0}$ half cone angle 


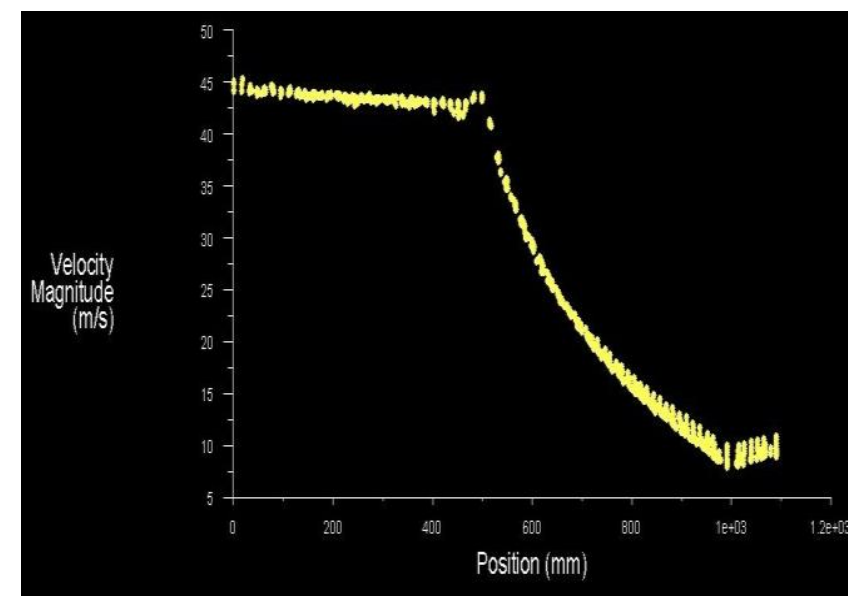

Fig 7 Contours of static pressure for diffuser of $7^{0}$ half cone angle

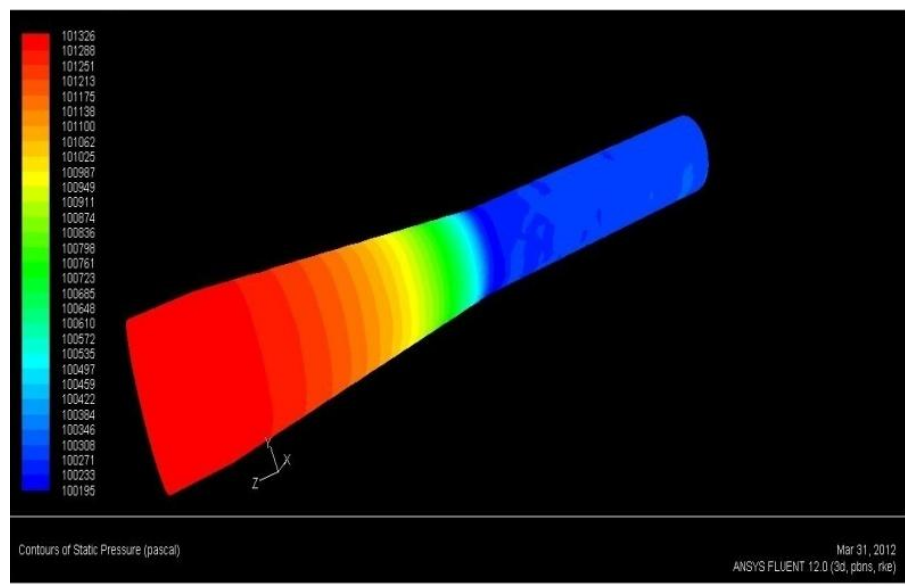

Fig 9 Contours of turbulent dissipation rate for diffuser of $7^{0}$ half cone angle

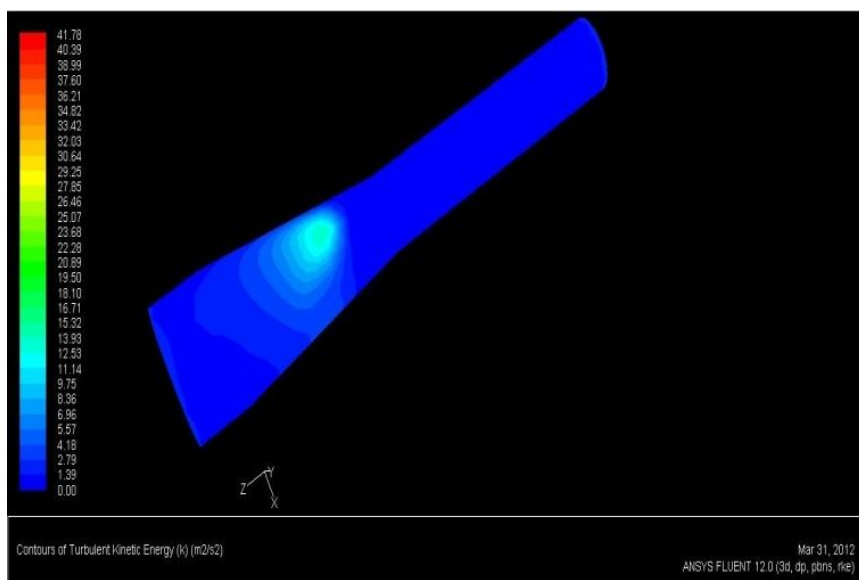

Fig 11 Contours of turbulent kinetic energy for diffuser with $7^{0}$ half cone angle

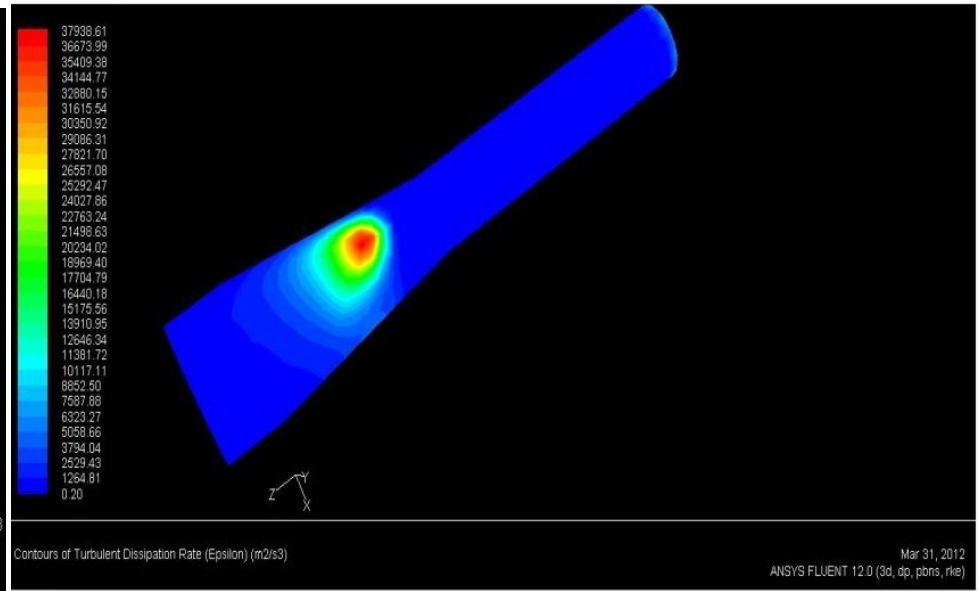

Fig 8 Contours of velocity magnitude for diffuser of $7^{0}$ half cone angle

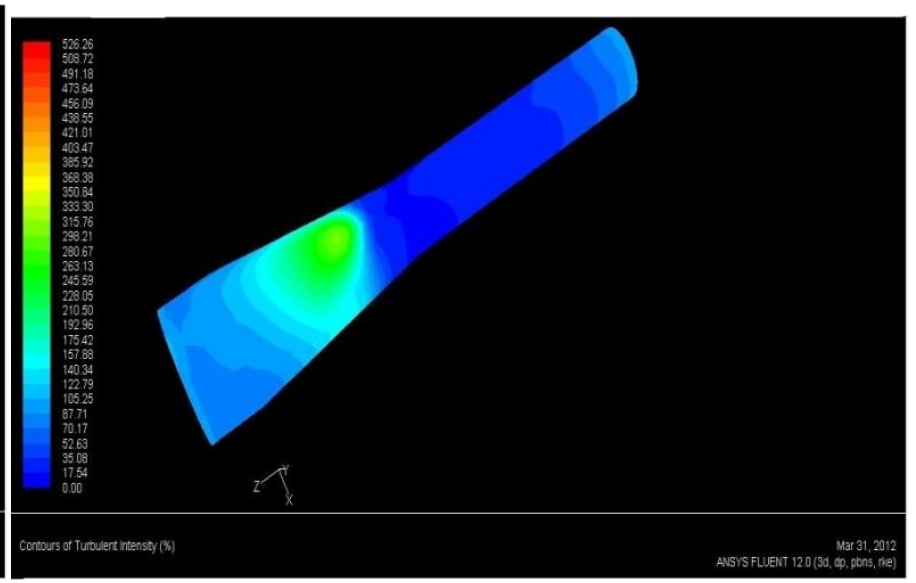

Fig 10 Contours of turbulent intensity for diffuser of $7^{0}$ half cone angle

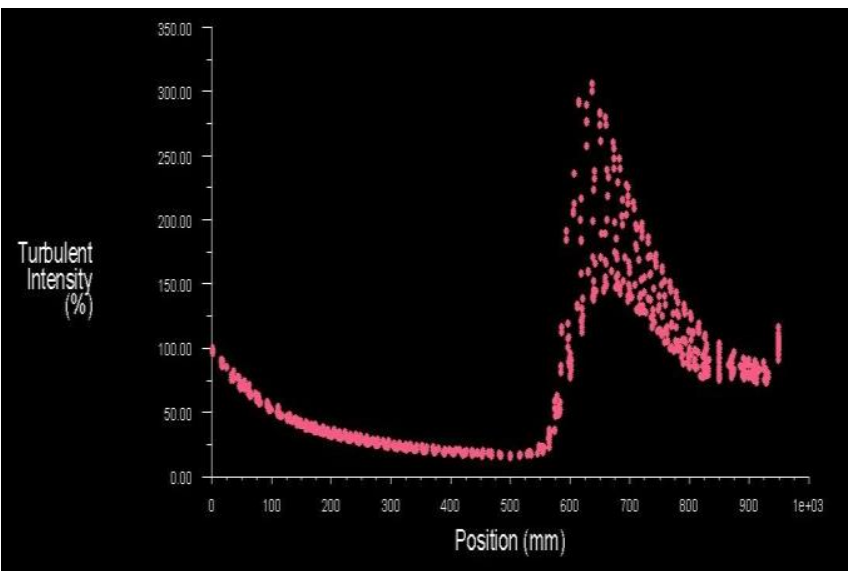

Fig 12 Variation of static pressure along the length for diffuser of $7^{0}$ half cone angle 


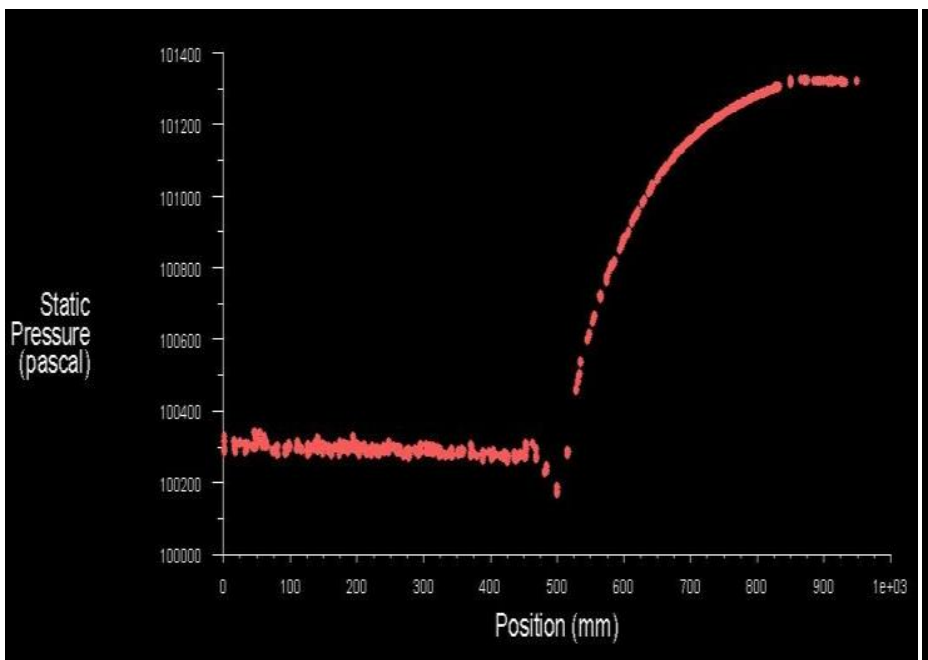

Fig 13 Variation of velocity magnitude along the length for diffuser of $7^{0}$ half cone angle

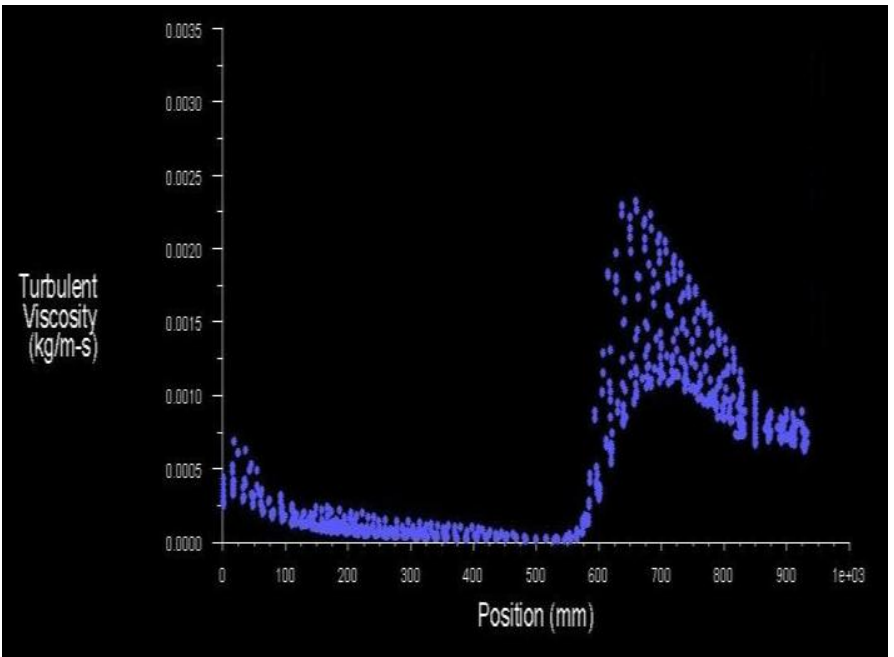

Fig 14 Variation of turbulent intensity along the length for diffuser of $7^{0}$ half cone angle

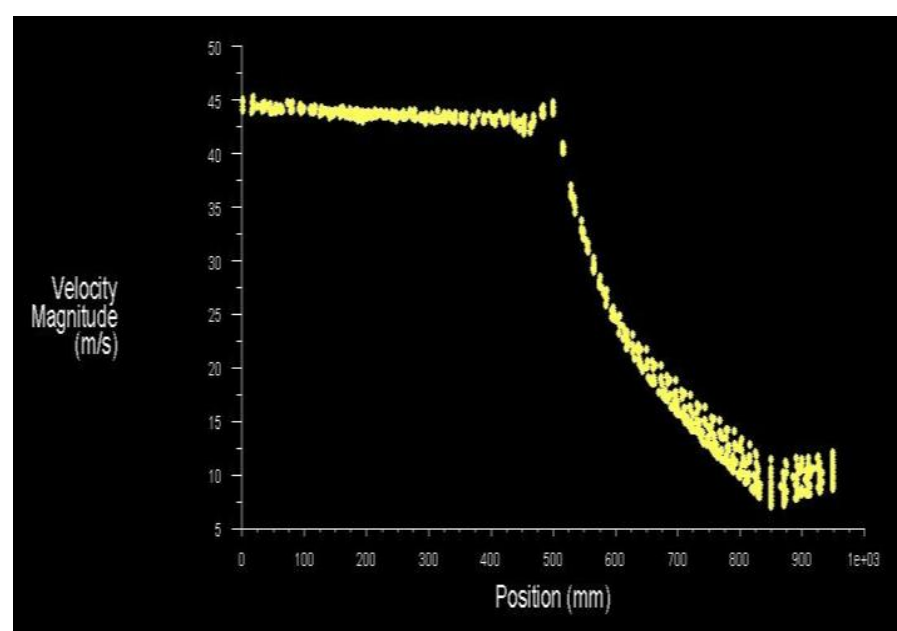

Fig 15 Variation of turbulent viscosity along the length for diffuser of $7^{0}$ half cone angle

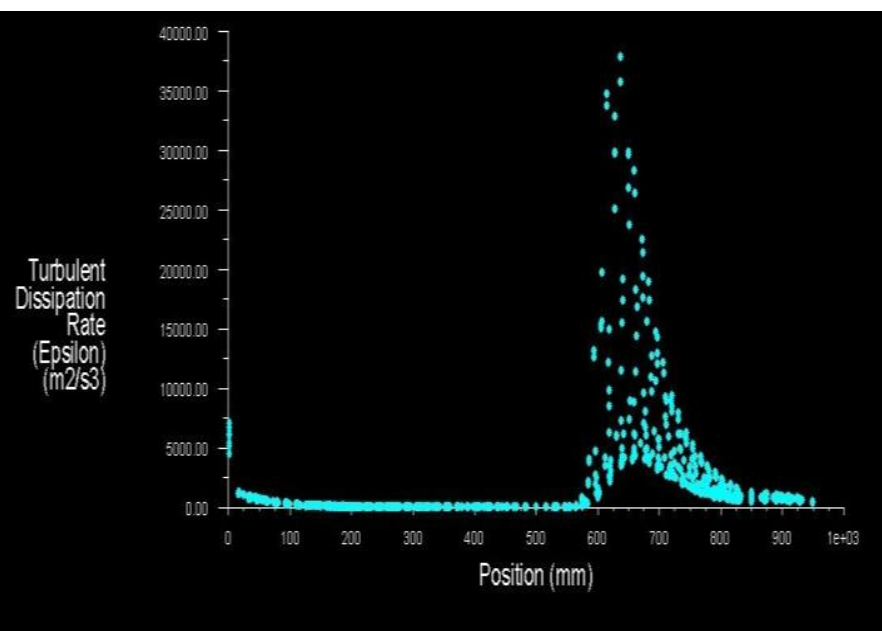

Fig 16 Variation of turbulent dissipation rate along the length for diffuser of $7^{0}$ half cone angle

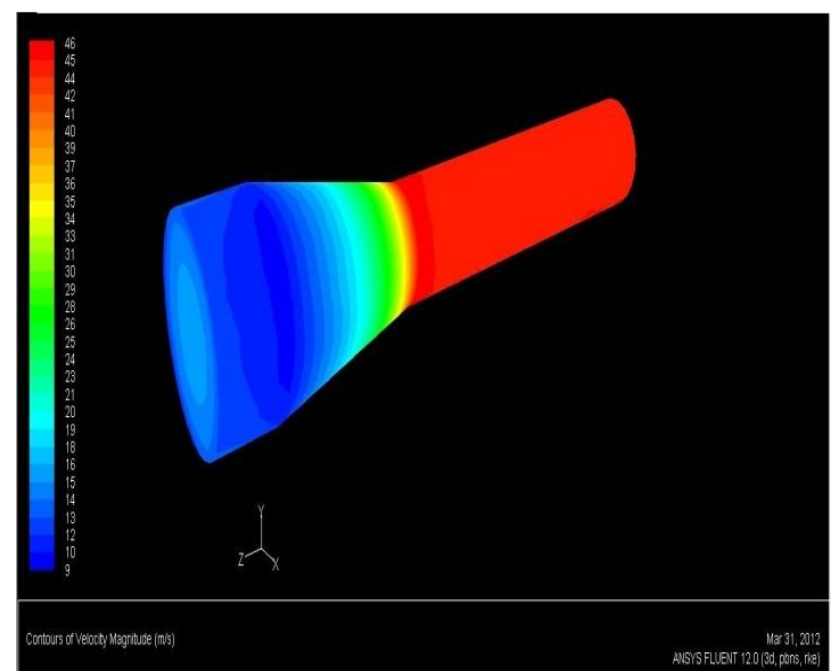

Fig 17 Contours of static pressure for diffuser with $10^{\circ}$ half cone angle

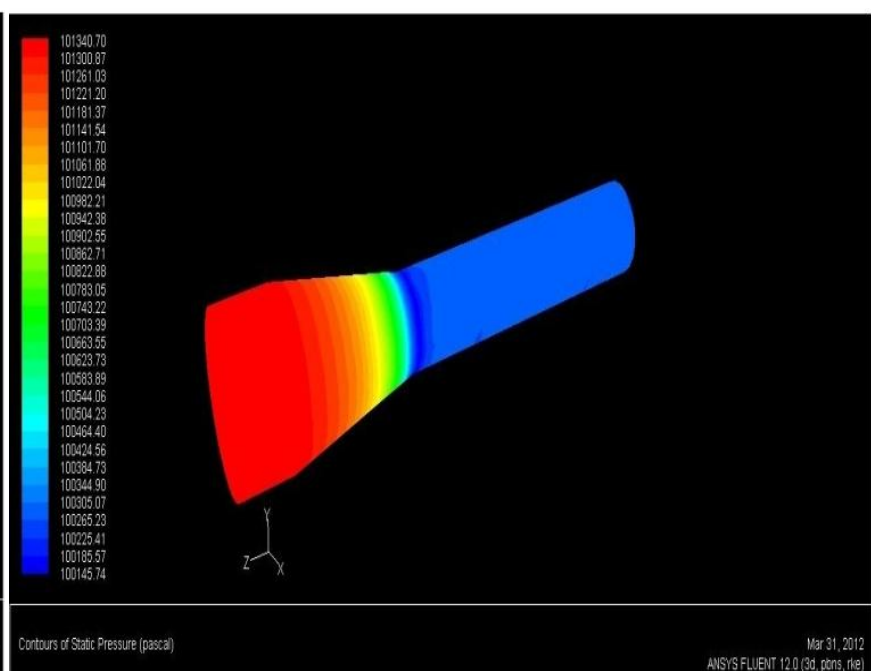

Fig 18 Contours of velocity magnitude for diffuser with $10^{0}$ half cone angle 


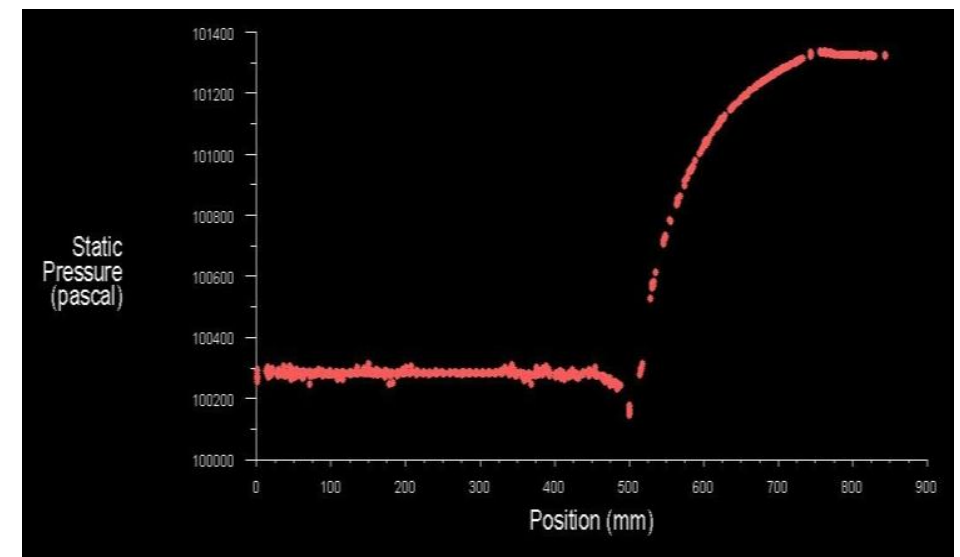

Fig 19 Variation of static pressure along the length for diffuser of $10^{\circ}$ half cone angle

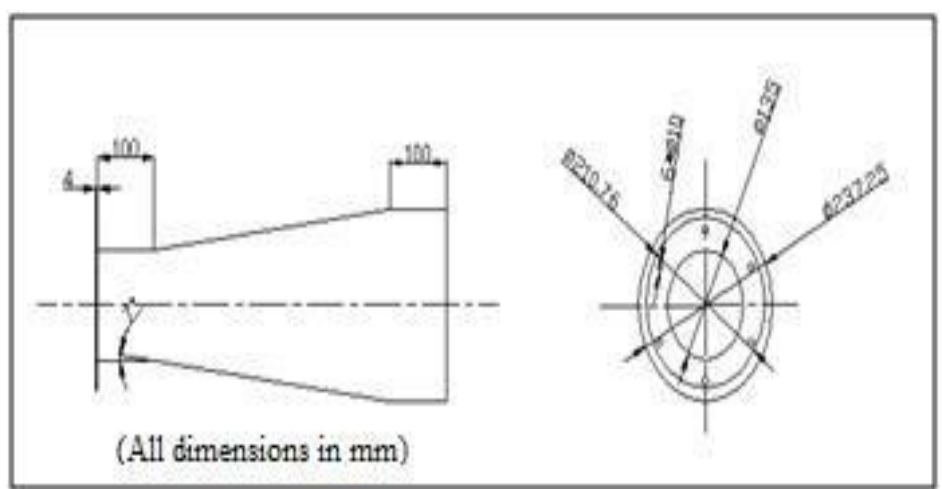

Fig 20 Dimensions of the fabricated diffuser

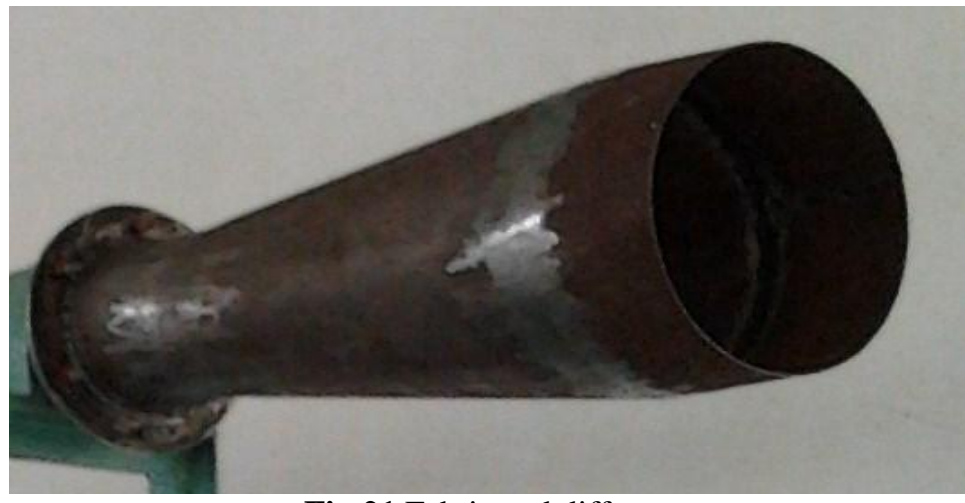

Fig 21 Fabricated diffuser

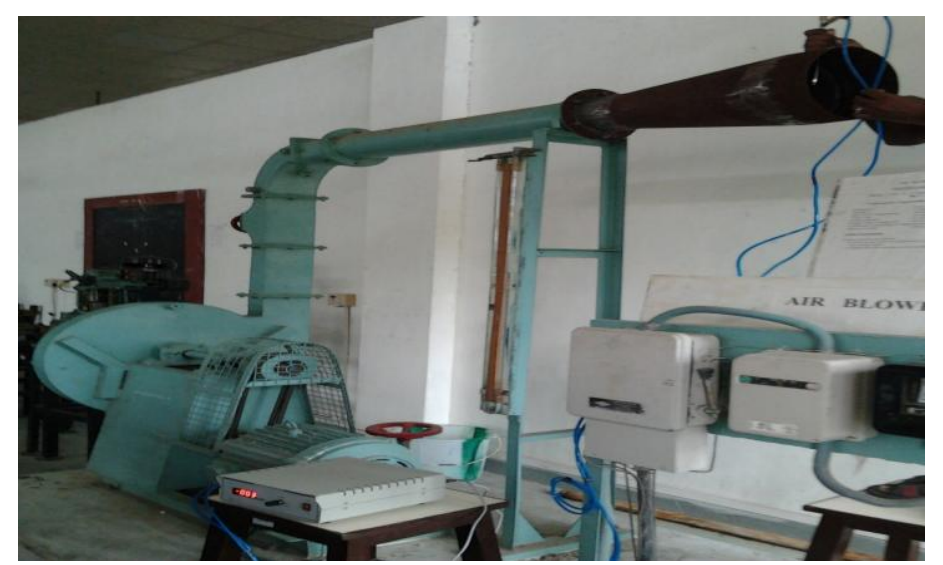

Fig 22 Experimental Set up 


\section{REFERENCES}

[1]. Kouichi Ishizaka, Susumu Wakazono (2003) "CFD Studies of Industrial Gas Turbine Exhaust Diffusers", Proceedings of the International Gas Turbine Congress Tokyo 2003.

[2]. SN Singh, V. Seshadri, K. Saha, K. K Vempati and S. Bharani (2006) "Effect of inlet swirl on the performance of annular diffusers having the same equivalent cone angle", Proc. Inst. Mech. Engineer, Part G, Journal of Aerospace Engineering Vol. 220, No.2, pp.129-143.

[3]. SJ Kline, DE Abbott, RW Fox (1959)“Optimum design of straight-walled diffusers", ASME Journal of Basic Engineering 91 321-330.

[4]. Bhaskharone, E.A (1991) "Finite-element analysis of turbulent flow in annular exhaust diffuser of gas turbine engines", ASME Transactions Journal of Fluid Engineering. [5]. A.C. Benim, W. Zinser (1985)“'Investigation into the finite element analysis of confined turbulent flows using a $\kappa$ $\varepsilon$ model of turbulence", Computer Methods in Applied Mechanics and Engineering, Volume 51, Issues 1-3, Pages 507-523.

[6]. Azad, R.S and Kassab, S.Z (1989) "Turbulent flow in conical diffuser: overview and implications", Physics fluids A 1 (3), 564-573.

[7]. V. Vassiliev, S. Irmisch, S. Florjancic (2002) "CFD Analysis of Industrial Gas Turbine Exhaust Diffusers", ASME Turbo Expo 2002 pp. 995-1013.

[8]. Okwuobi, P.A.C and Azad, R.W (1973) "Turbulence in a conical diffuser with fully developed flow at entry", Journal of Fluid Mechanics.

[9]. Hoffman, JA, Gonzalez.G (1984) "Effects of smallscale, high intensity inlet turbulence on flow in a twodimensional diffuser", ASME, Transactions, Journal of Fluids Engineering. Vol. 106, pp. 121-124.

[10]. Armfield, S.W and Fletcher, C.A.J (1989) "Comparison of $\mathrm{k}-\mathrm{e}$ and algebraic Reynolds stress models for swirling diffuser flow", International journal of Numerical methods in fluids.

[11]. B. K. Sultanian, S. Nagao and T. Sakamoto (1999) "Experimental and Three-Dimensional CFD Investigation in a Gas Turbine Exhaust System", J. Eng. Gas Turbines Power Volume 121, Issue 2, 364 Investigation in a Gas Turbine Exhaust system", J. Eng. Gas Turbines Power Volume 121, Issue 2, 364.

[12]. N.V. Mahalakshmi, G. Krithiga, S. Sandhya, J. Vikraman and V. Ganesan (2007) "Experimental investigations of flow through conical diffusers with and without wake type velocity distortions at inlet", Experimental Thermal and Fluid Science Volume 32, Issue 1, Pages 133-157

\section{BIOGRAPHIES}

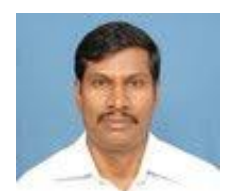

Dr.R.Prakash, Assistant Professor, Department of Mechanical Engineering SSN College of Engineering Kalavakkam, Chennai 603110

Tel: 00919962422296

Email: prakashr@ssn.edu.in

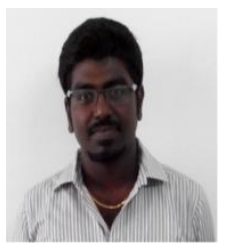

D.Christopher, M E Energy Engineering Student, Department of Mechanical Engineering, SSN College of Engineering, Kalavakkam, Chennai 603110

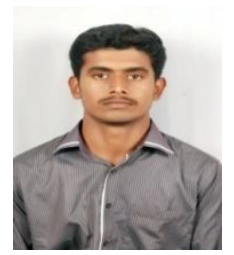

K.Kumarrathinam, M E Energy Engineering Student, Department of Mechanical Engineering, SSN College of Engineering, Kalavakkam, Chennai 603110 